\title{
A feature extraction method based on VMD and RDT and its application in engine crankshaft bearing fault
}

\author{
Gang Ren ${ }^{1}$, Jide $\mathrm{Jia}^{2}$, Jianmin $\mathrm{Mei}^{2}$, Xiangyu Jia ${ }^{1}$, Jiajia Han $^{1}$ \\ 1 Postgraduate Training Brigade, Army Military Transportation University, Tianjin 300161, China; \\ jjxyrengang@163.com (G.R.); kittyyoyo_jxy@126.com (X.J.); 474689816@qq.com (J.H.) \\ 2 Military Vehicle Department, Army Military Transportation University, Tianjin 300161, China; \\ jide@ustc.edu (J.J.); jjxyrg@163.com (J.M.) \\ * Correspondence: jjxyrengang@163.com or jide@ustc.edu ; Tel:+86-022-84658841
}

\begin{abstract}
The vibration signal of the engine contains strong background noise and many kinds of modulating components, which is difficult to diagnose. Variational mode decomposition (VMD) is a recently introduced adaptive signal decomposition algorithm with a solid theoretical foundation and good noise robustness compared with empirical mode decomposition (EMD). VMD can effectively avoid endpoint effect and modal aliasing. However, VMD cannot effectively eliminate the random noise in the signal, so the random decrement technique is introduced to solve the problem. Based on the crankshaft bearing fault simulation experiment, the four kinds of wear state vibration signals are decomposed by VMD, and the modal components with smaller permutation entropy are selected as fault components. Then the fault component is processed by the random decrement technique, and the Hilbert envelope spectrum of the fault component is obtained. Compared with the fault feature extraction method based on EMD and EEMD, the feature extraction results of the proposed method are better than those of the above two methods. The simulation analysis and the simulation test of the crankshaft bearing fault verify the effectiveness of the proposed method.
\end{abstract}

Keywords: variational mode decomposition; random decrement technique; crankshaft bearing; engine; feature extraction

\section{Introduction}

The engine is a complex mechanical device, with the characteristics of multi-source, multi moving parts, and complex work. The engine has both rotational and reciprocating motion. Vibration signals are fully used in fault diagnosis because of their convenience [1-2]. The vibration signal of engine is composed of multi-component complex signals, and its amplitude varies with time. For the complex multi-component signal, it is usually necessary to decompose it into a number of single-component AM-FM signals, and each component is analyzed to extract amplitude and frequency information.

In order to solve the above problems, Huang et al. introduced an adaptive signal processing technique called empirical mode decomposition (EMD) [3-4], which has demonstrated outstanding performance in dealing with nonlinear and nonpstationary signals. This technique has been applied in many fields, such as biomedical image analysis [5], fault diagnosis of rolling element bearings [6], signal de-noising [7-9], and voice signal analysis [10]. But there are still some problems for EMD, such as endpoint effect and modal aliasing [11]. Wu [12] proposed Ensemble Empirical Mode Decomposition (EEMD). Different white noises are added to the 
original signal for EMD, and multiple decomposition results are averaged to obtain the final Intrinsic Mode Function (IMF). The high frequency modulation information in the signal can be separated very well, and the modal aliasing of EMD is well suppressed [13]. However, the white noise can easily lead to the reconstruction error for EEMD.

In recent years, Konstantin, Dragomiretskiy et al. proposed variational modal decomposition [14], which is essentially composed of several adaptive Wiener filters and has good noise robustness. Compared with EMD, VMD has strong mathematical theory basis. So it can effectively alleviate or avoid a series of problems that exist in EMD, and has higher operation efficiency. VMD is widely used in various engineering fields [15-18]. An X.L. et al. applied VMD to the bearing fault diagnosis of the wind turbine, and realized the effective discrimination of the bearing fault [19]. By combining VMD with detrended fluctuation analysis (DFA), Liu et al. successfully extracted gear fault characteristics [20]. By combining VMD with independent component analysis (ICA), Yao et al. successfully separated the piston knock and combustion noise of the engine [21]. However, there is nothing VMD can do about the random noise in the signal.

In order to solve the problem, the random decrement technique (RDT) is introduced in this paper. Random decrement technique is a method of identification of modal parameters, which is first proposed by Cole [22-23] in 70s. The basic concept of RDT is to assume a system with stationary random excitation, and its response is the superposition of both deterministic and random responses. The deterministic response is separated from the random response, and the random response is eliminated by using the statistical mean method. Finally, a deterministic free attenuation signal is obtained by filtering. RDT has been widely used in many fields such as vibration modal analysis [24], and structural damage detection [25].

In this paper, a new fault feature extraction method for engine crankshaft bearing is proposed based on the variational mode decomposition and random decrement technique. First, the VMD is used to decompose the fault signal into several modal components, and the fault components are selected according to the permutation entropy. Then the fault component is processed by the random decrement technique and the Hilbert envelope spectrum is calculated to extract the fault features. The effectiveness of the proposed method is verified by simulation analysis and the simulation experiment of crankshaft bearing fault.

\section{Variational Mode Decomposition}

The VMD algorithm defines the intrinsic mode function as a non-stationary AM-FM signal. The intrinsic mode is considered as follows:

$$
u_{k}(t)=A_{k}(t) \cos \left(\varphi_{k}(t)\right)
$$

Where the phase $\varphi_{\mathrm{k}}(t)$ shall satisfy the following condition: $\varphi_{k}(t)^{\prime} \geqslant 0$; the envelope line $A_{k}(t)$ should satisfy the following condition: $A_{k}(t) \geqslant 0$; the instantaneous frequency $\omega_{k}(t)$ should satisfy the following condition: $\omega_{k}(t)=\varphi_{k}(t)^{\prime} . A_{k}(t)$ and $\omega_{k}(t)$ change slowly, and $\varphi_{k}(t)$ changes more rapidly.

The Hilbert transform is performed for each modal function $u_{k}(t)$, and exponential correction is applied to obtain $\mathrm{K}$ modal functions. Then the frequency spectrum of the modal function is corrected to the estimated central frequency, and the bandwidth of the modal component is calculated by using Gauss smoothing. The variational constraint problem can be defined as follows:

$$
\min _{\left\{u_{k}\right\},\left\{w_{k}\right\}}\left\{\sum_{k=1}^{K} \| a_{t}\left\{\left[\left(\delta(t)+\frac{j}{\pi t}\right] * u_{k}(t)\right\} e^{j \omega_{k} t} \|_{2}^{2}\right\} \text { s.t. } \sum_{k=1}^{K} f(t)\right.
$$


Where $u_{k}$ is the modal component, $\omega_{k}$ is the central frequency for the modal component, $\delta(t)$ is the unit pulse function, and * is the convolution symbol.

In the VMD algorithm, the secondary penalty factor and the Lagrangian multiplication operator are used. Then, the alternating direction method is introduced. $u_{k}{ }^{n+1}, \omega_{k}{ }^{n+1}$, and $\lambda^{n+1}$ are constantly updated, so that the optimal solution of the variational constraint problem can be solved. The expression for the modal component $u_{k}^{n+1}$ is

$$
u_{k}{ }^{n+1}=\underset{u_{k} \in X}{\operatorname{argmin}}\left\{\alpha \| a_{t}\left\{\left[\left(\delta(t)+\frac{j}{\pi t}\right]^{*} u_{k}(t)\right\} e^{j \omega_{k} t}\left\|_{2}^{2}+\right\| f(t) \quad \sum_{i} u_{i}(t)+\frac{\lambda(t)}{2} \|_{2}^{2}\right\}\right.
$$

where $\alpha$ is the penalty factor, and $\lambda$ is the Lagrange multiplier.

The expression for the modal component $u_{k}{ }^{n+1}$ in frequency domain is

$$
\hat{u}_{k}^{n+1}(\omega)=\left(\hat{f}(\omega)-\sum_{i} \hat{u}_{i}(\omega)+\frac{\hat{\lambda}(\omega)}{2}\right) \frac{1}{1+2 \alpha\left(\omega \omega_{k}\right)^{2}}
$$

Where $\omega_{k}$ is the center of the modal component power spectrum. The Wiener filter is introduced, which makes the VMD algorithm have better noise robustness.

Similarly, the expression for the central frequency $\omega_{k}{ }^{n+1}$ is

$$
\omega_{k}^{n+1}(\omega)=\frac{\int_{0}^{\infty} \omega\left|\hat{u}_{k}(\omega)\right|^{2} d \omega}{\int_{0}^{\infty}\left|\hat{u}_{k}(\omega)\right|^{2} d \omega}
$$

The stopping condition of the iteration is

$$
\sum_{k=1}^{K}\left\|\hat{u}_{k}^{n+1} \hat{u}_{k}^{n}\right\|_{2}^{2} /\left\|\hat{u}_{k}^{n}\right\|_{2}^{2}<e
$$

According to the previous derivation, we get the complete algorithm for VMD, summarized in algorithm 1.

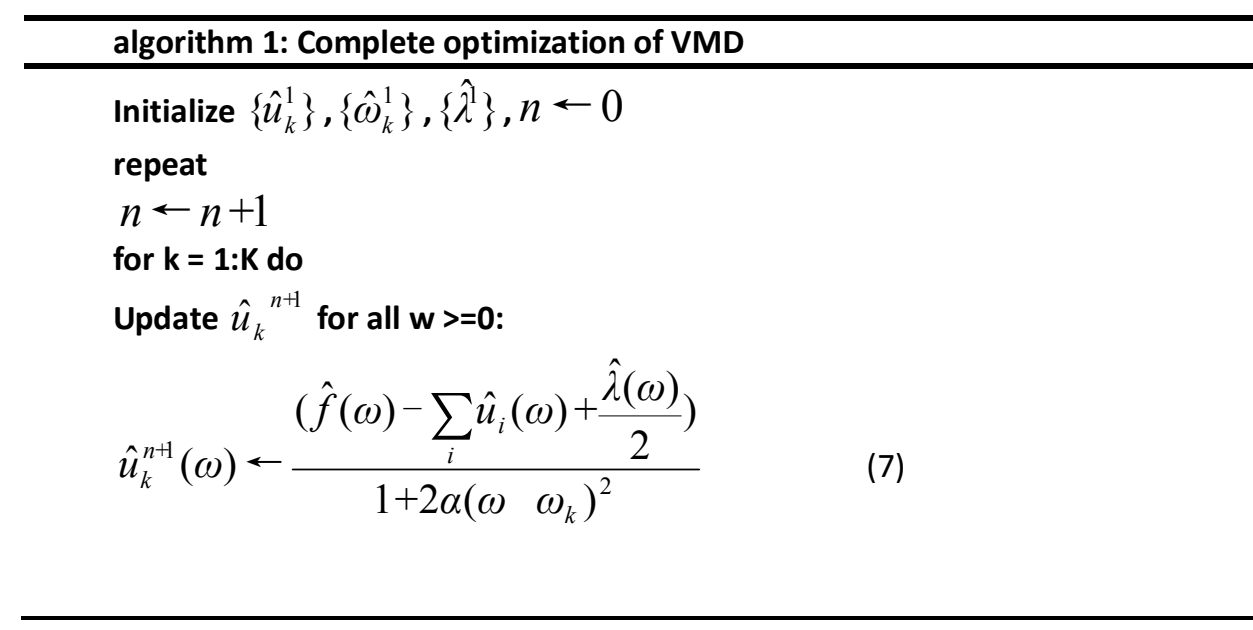




\section{Update $\mathbf{w}_{\mathrm{k}}$ :}

$\omega_{k}^{n+1}(\omega) \leftarrow \frac{\int_{0}^{\infty} \omega\left|\hat{u}_{k}(\omega)\right|^{2} d \omega}{\int_{0}^{\infty}\left|\hat{u}_{k}(\omega)\right|^{2} d \omega}$

\section{end for}

Dual ascent for all $w>=0$ :

$$
\hat{\lambda}^{n+1}(\omega) \leftarrow \hat{\lambda}^{n}(\omega)+\beta\left[\hat{f}(\omega) \sum_{k=1}^{K} \hat{u}_{k}^{n+1}(\omega)\right]
$$

\section{until convergence:}

$$
\sum_{k=1}^{K}\left\|\hat{u}_{k}^{n+1} \quad \hat{u}_{k}^{n}\right\|_{2}^{2} /\left\|\hat{u}_{k}^{n}\right\|_{2}^{2}<e
$$

The VMD algorithm is a linear transformation, so the signal can be reconstructed. The reconstructed signal can be represented as:

$$
\hat{f}(t)=\sum_{k=1}^{K} \hat{u}_{k}
$$

Where $\hat{u}_{k}$ is the final modal component, after the iteration is stopped.

\section{Random Decrement Technique}

RDT can be used to describe the impulse response of the system, and its advantage is that it can extract free impact response from the stationary random response in the system. The core is to assume that a system is subjected to stationary random excitation, and the response is the superposition of deterministic response determined by the initial condition and random response determined by the initial external load. Under the same initial conditions, stationary random response is divided into several sections, and the general mean of the intercept segments is calculated, so as to extract the free attenuation response.

The response signal is divided into $L$ segments, and each segment of the signal is expressed as $x_{i}(t)$, whose length is $\tau$. Each response signal has the same trigger value, and the trigger value can be expressed as

$$
x_{i}\left(t_{i}\right)=x_{s}=\text { const }, i=1,2, \cdots, L
$$

The overall mean of the $L$ segment signal is calculated, and the random decrement function can be expressed as:

$$
x(\tau)=\frac{1}{L} \sum_{i=1}^{L} x_{i}\left(t_{i}+\tau\right)
$$

where $x_{i}\left(t_{i}\right)=x_{s}, i=1,2, \cdots, L$.

\section{Proposed Method}

Vibration signal analysis is usually used for condition monitoring and fault diagnosis. However, due to the complex structure of engines, the vibration signals of engines are usually multi-component, non-stationary and non-Gauss. In addition, there is a large amount of background noise in the vibration signals of engines. Therefore, it is very difficult to extract 
fault characteristics from the vibration signals of engines.

The permutation entropy is a new method of mutation detection, which mainly aims at the spatial characteristics of time series [26-27]. The permutation entropy is very simple in theory and has good noise robustness. Besides, the permutation entropy also has a high resolution, and the output results are very intuitive. The permutation entropy reflects the random degree of the signal. In other words, the smaller the permutation entropy, the more fault information the time series has. Therefore, the permutation entropy is used to select the fault component in this paper.

VMD is a recently proposed signal decomposition method, which is essentially composed of a number of adaptive Wiener filters, and has good noise robustness. Compared with the EMD method, the VMD method has a solid mathematical theoretical foundation, and can effectively alleviate or avoid a series of shortcomings in the EMD method. To verify the efficiency of the proposed method, several experiments on engine crankshaft bearing faults are performed. The detailed experimental scheme is shown in Figure 1. Firstly, a single channel vibration signal $x(t)$, which is a wearing fault of the engine crankshaft bearing, is collected by the acceleration sensor vertically fixed on the engine block. Secondly, the collected vibration signal $x(t)$ is decomposed into several modal components by VMD method. Thirdly, the permutation entropy of each modal component is calculated respectively, and the modal components with smaller permutation entropy are selected as the fault components. Lastly, the fault components are processed by the random decrement technique and the Hilbert envelope spectrum is calculated to extract the fault features.

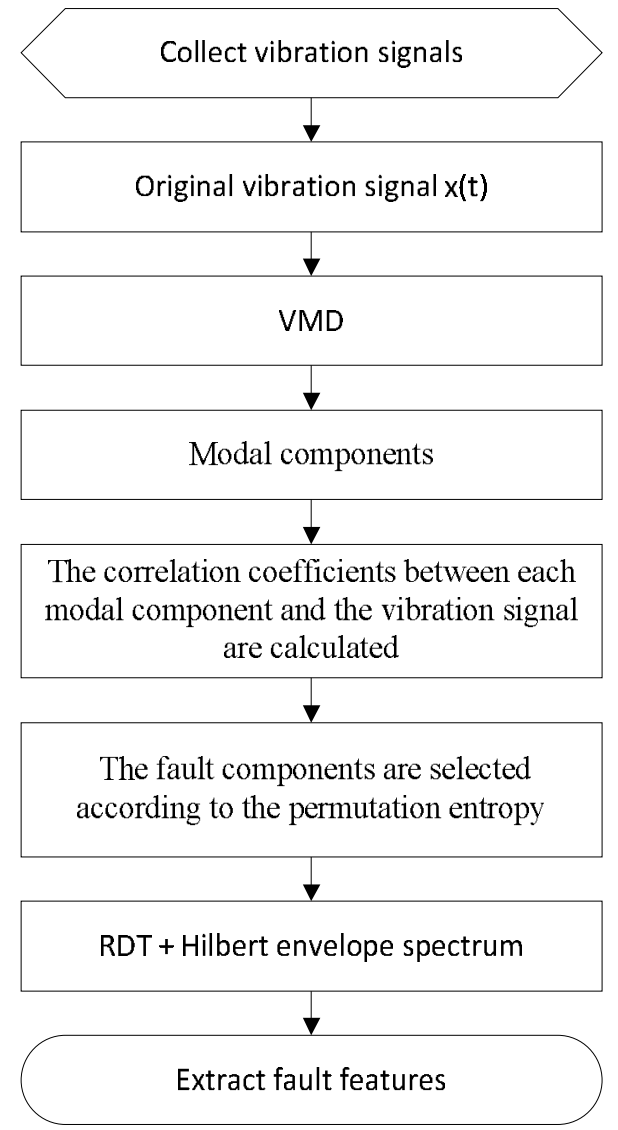

Figure 1. Detailed experimental scheme. 


\section{Experimental Results}

\subsection{Simulation}

Engine crankshaft bearing consists of two bearing bushings. When the crankshaft bearing has a wear fault, it is mainly under attack by the rotation of the crankshaft and the ignition of the cylinder. Therefore, the characteristic frequency of engine crankshaft bearing fault is mainly the rotation frequency of engine crankshaft and the ignition frequency of engine. Because of the complex structure of engine, the number of vibration excitation source is large, and the vibration source signal is modulated by several components. Therefore, the established simulation signal must be multi-component and non-Gauss. The simulation signal is as follows:

$$
\begin{aligned}
c(t)=0.5 \sin \left(2 \pi f_{0}\right)+0.15 \sin \left(4 \pi f_{0}\right)+0.05 \sin \left(6 \pi f_{0}\right) \\
s(t)=e^{-B t} \cos \left(2 \pi f_{n} t\right) \\
x(t)=s(t+T)+c(t)+e(t) \\
B=2 \pi f_{n} \xi
\end{aligned}
$$

Where $c(t)$ is a low frequency stable signal associated with the rotation frequency $f_{0}$ and its harmonic component of the crankshaft, $s(t)$ is the periodic impact component produced by the ignition of the cylinder, $T$ is the pulse period, $f_{n}$ is the resonance frequency of the engine itself, $B$ is the attenuation coefficient of the engine, $\xi$ is the damping ratio of engine vibration and $e(t)$ is Gauss white noise. The parameters are set as follows:

$f_{0}=30 \mathrm{~Hz}, f_{n}=100 \mathrm{~Hz}, \xi=0.0198194, T=0.01 \mathrm{~s}$.

The sampling frequency is $20000 \mathrm{~Hz}$, and the number of sampling points is 16384 . The time-domain and frequency domain waveform of the noisy simulation signal is shown in Figure 2. As you can see in Figure 2, the signal is disturbed by the background noise. The crankshaft rotation frequency and the periodic impact component are not obvious, and only the resonance frequency of the engine can be identified.

(a) The time domain w aveform

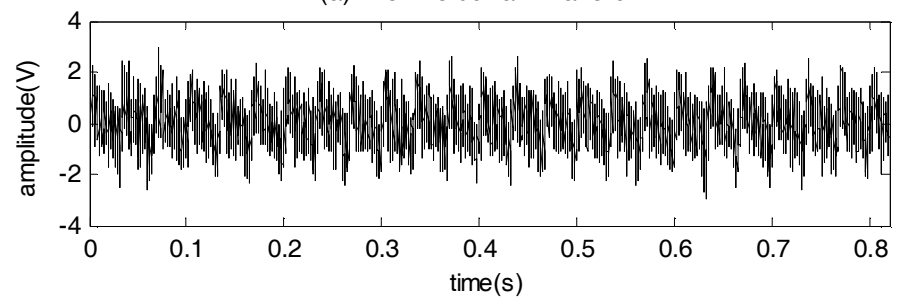

(b) The frequency domain w aveform

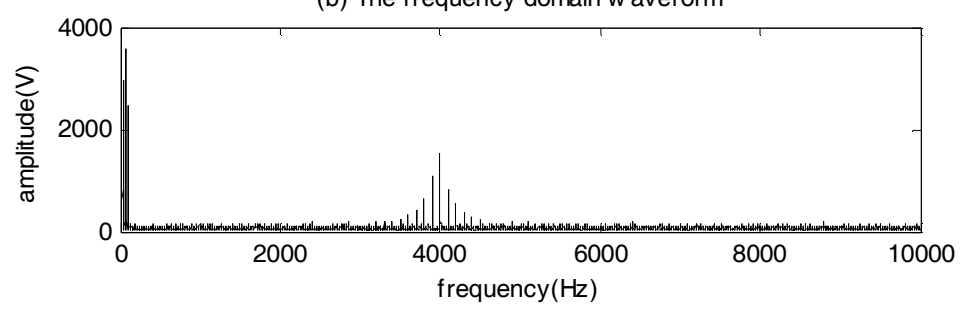

Figure 2. The noisy simulation signal.

According to the composition of the simulation signal, the modal number $\mathrm{K}$ is set to 4 . $\mathrm{VMD}$ is used to decompose the simulation signal into 4 modal components, as shown in Figure 3. As you can see from Figure 3, there are some impact components in the signal, but there is no more fault information. 

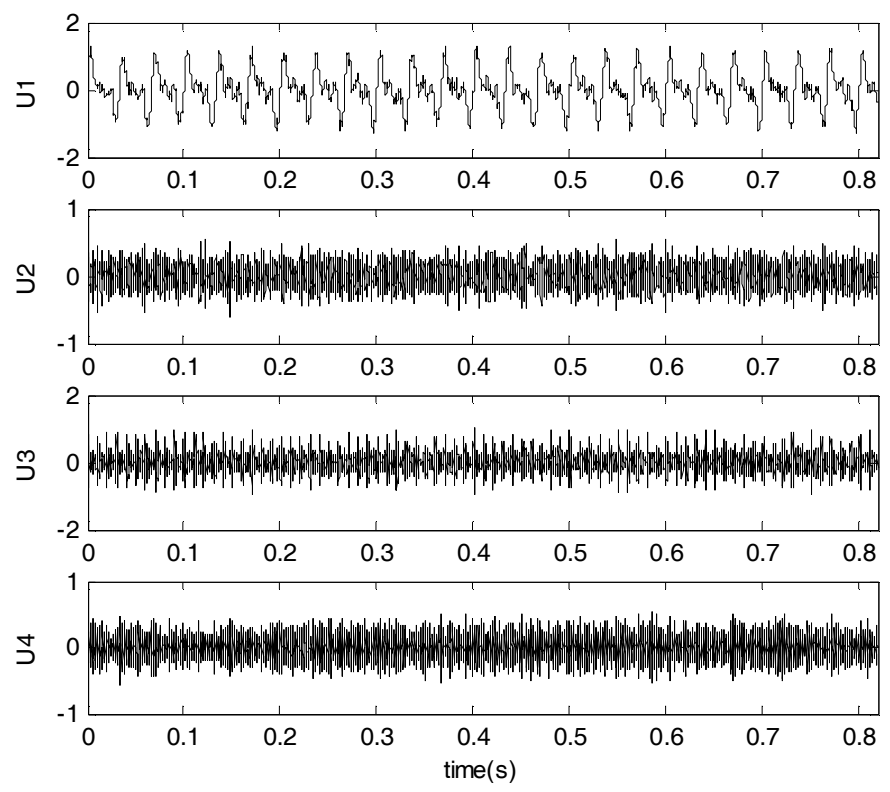

Figure 3. BLIMFs decomposed by the VMD method.

In order to find out the modal components containing rich fault information from the decomposition results, the permutation entropy of the modal components is calculated respectively, as shown in Table 1.

Table 1. The permutation entropy of the components.

\begin{tabular}{ccccc}
\hline Modal component & U1 & U2 & U3 & U4 \\
\hline Permutation entropy & 0.335 & 0.551 & 0.364 & 0.726 \\
\hline
\end{tabular}

As can be seen from table 1, the permutation entropy of component $\mathrm{U} 1$ and $\mathrm{U} 3$ is the smallest, that is, the two components contain the most abundant fault information. Therefore, U1 and U3 are selected as fault components. The Hilbert envelope spectrum of U1 and U3 is shown in Figure 4.

(a) U1

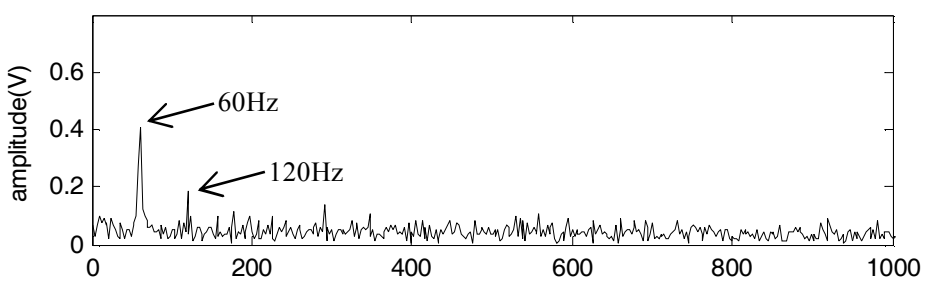

(b) U3

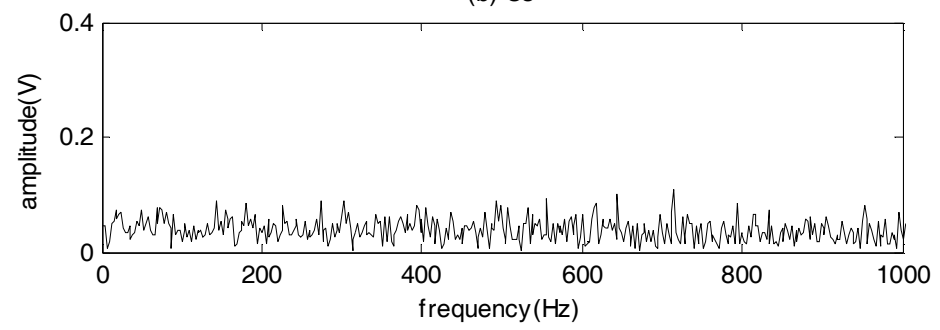

Figure 4. The Hilbert envelope spectrum of U1 and U3. 
As can be seen from Figure 4, in the envelope spectrum of the component U1, 2 times the rotation frequency $60 \mathrm{~Hz}$ and 4 times the rotation frequency $120 \mathrm{~Hz}$ appear. There are many interference frequencies in the envelope spectrum of the component U3, and the fault features cannot be extracted effectively. In order to further extract the fault features, the fault components are processed by RDT. The trigger value is set to 1.5 times the standard deviation and the Hilbert envelope spectrum of the fault component is calculated, as shown in Figure 5.

(a) U1

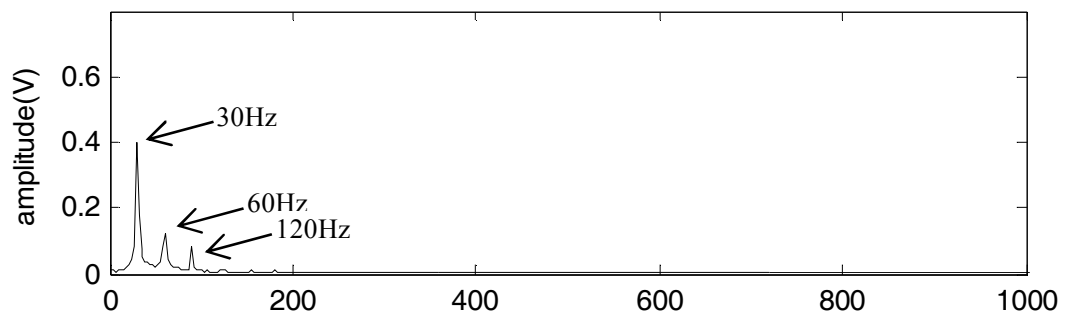

(b) U3

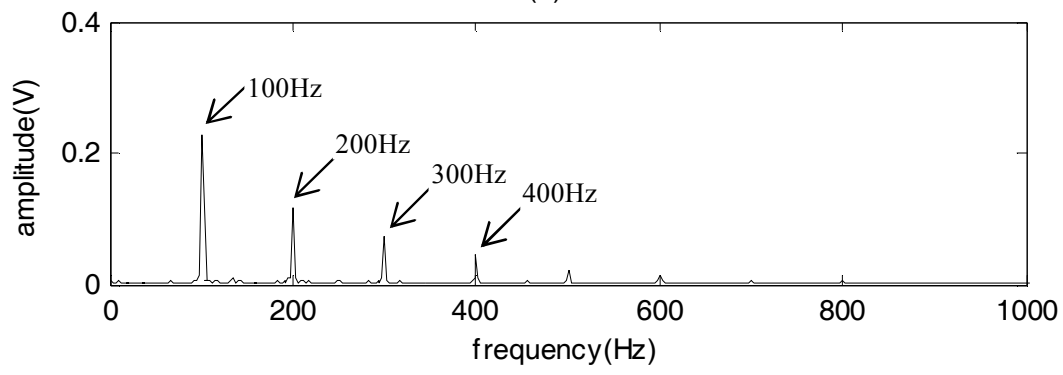

Figure 5. The Hilbert envelope spectrum of the fault components after RDT.

As can be seen from Figure 5, after the fault components are processed by RDT, the rotation frequency $30 \mathrm{~Hz}, 2$ times the rotation frequency $60 \mathrm{~Hz}$, and 3 times the rotation frequency $90 \mathrm{~Hz}$ appear in the envelope spectrum of $\mathrm{U} 1$. The ignition frequency $100 \mathrm{~Hz}, 2$ times the ignition frequency $200 \mathrm{~Hz}, 3$ times the ignition frequency $300 \mathrm{~Hz}$ and 4 times the ignition frequency $400 \mathrm{~Hz}$ appear in the envelope spectrum of U3. Therefore, the fault frequency and its harmonics are successfully extracted, that is, the proposed method has successfully extracted the fault features from the simulation signal.

\subsection{Experiment Condition}

The structure of engine is complex and the working environment is abominable. As a result, it is prone to malfunction. The crankshaft bearing is located inside the engine, so it is difficult to diagnose the fault. In this paper, vibration signals are collected from the vibration sensors on the experimental stand, as shown in Figure 6. The basic parameters of the vibration sensor are shown in table 2. The engine on the experimental stand is Cummins 6BT diesel engine, and its parameters are shown in table 3. 


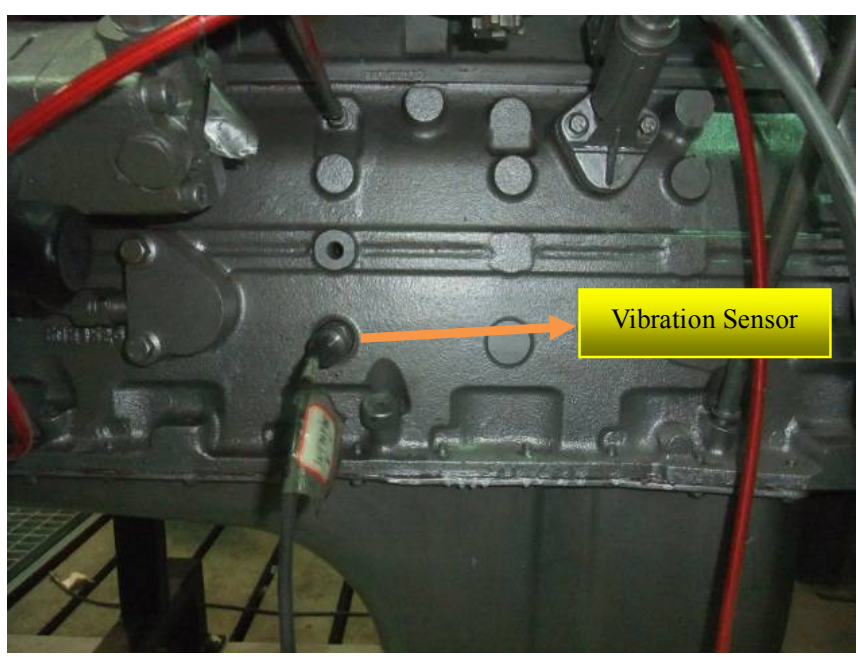

Figure 6. Measuring position of vibration sensor.

Table 2. Vibration sensor parameters

\begin{tabular}{|c|c|c|c|c|c|c|c|c|}
\hline Model & Sensitivity & \multicolumn{2}{|c|}{$\begin{array}{l}\text { Frequency range } \\
\qquad( \pm 3 \mathrm{~dB})\end{array}$} & Range & Resolution & $\begin{array}{c}\text { Temperature } \\
\text { range }\end{array}$ & Weight & $\begin{array}{c}\text { Output } \\
\text { connect } \\
\text { or }\end{array}$ \\
\hline $603 C 01$ & $100 \mathrm{mV} / \mathrm{g}$ & \multicolumn{2}{|c|}{$0.5 \mathrm{~Hz}-10 \mathrm{KHz}$} & $\pm 50 \mathrm{~g}$ & $350 \mu \mathrm{g}$ & $-54-121^{\circ} \mathrm{C}$ & $51 \mathrm{~g}$ & Top \\
\hline \multicolumn{2}{|c|}{ Engine type } & 6BT5.9-G2 & \multicolumn{2}{|c|}{ Fuel type } & $\begin{array}{c}\text { Diesel } \\
\text { oil }\end{array}$ & Type & \multicolumn{2}{|c|}{$\begin{array}{l}\text { Inline } 6 \\
\text { cylinders }\end{array}$} \\
\hline \multicolumn{2}{|c|}{$\begin{array}{l}\text { Rated power } \\
\text { (KW) }\end{array}$} & 118 & \multicolumn{2}{|c|}{ Compression ratio } & 17.5: 1 & $\begin{array}{l}\text { Ignition } \\
\text { sequence }\end{array}$ & \multicolumn{2}{|r|}{153624} \\
\hline \multicolumn{2}{|c|}{$\begin{array}{l}\text { Rated speed } \\
\text { (RPM) }\end{array}$} & 2600 & \multicolumn{2}{|c|}{$\begin{array}{c}\text { Continuous power } \\
(\mathrm{KW})\end{array}$} & 86 & $\begin{array}{l}\text { Maximum } \\
\text { torque }(\mathrm{N} \cdot \mathrm{m})\end{array}$ & \multicolumn{2}{|r|}{558} \\
\hline \multicolumn{2}{|c|}{$\begin{array}{c}\text { Radius }(\mathrm{mm}) \\
\times \text { Distance }(\mathrm{mm})\end{array}$} & $102 \times 120$ & \multicolumn{3}{|c|}{$\begin{array}{c}\text { Maximum torque speed } \\
(\mathrm{r} / \mathrm{min})\end{array}$} & 1600 & & \\
\hline
\end{tabular}

The fourth crankshaft bearings of Cummins EQ6BT engine are set with different clearance $(0.10 \mathrm{~mm}, 0.14 \mathrm{~mm}, 0.20 \mathrm{~mm}, 0.34 \mathrm{~mm})$ to simulate the normal, minor, moderate and severe wear of the connecting rod bearing. Vibration signals are collected on the left side of the fourth main bearings on the surface of the engine block. The sampling frequency is $20000 \mathrm{~Hz}$ and the sampling points are 4096 points.

Testing temperature is important when acquiring vibration signals. In the experiment, the temperature of cooling water is measured to reflect the internal temperature of engine. The temperature is controlled at 60-70 degrees $C$.

\subsection{Data Acquired}

The acquisition system is composed of collector, computer, sensor and connecting circuit, as shown in Figure 7. According to document [28], the optimum diagnostic speed of this type of engine is $1800 \mathrm{r} / \mathrm{min}$. In addition, four speeds were collected in the experiment: $800 \mathrm{r} / \mathrm{min}$, $1300 \mathrm{r} / \mathrm{min}, 1800 \mathrm{r} / \mathrm{min}$, and $2100 \mathrm{r} / \mathrm{min}$. The experiment proved that $1800 \mathrm{r} / \mathrm{min}$ is the most suitable for the fault diagnosis speed. Therefore, the acquisition system set the speed of the 
engine to $1800 \mathrm{r} / \mathrm{min}$. Through the theoretical calculation, the rotation frequency of the crankshaft is about $30 \mathrm{~Hz}$, and the engine ignition frequency is about $90 \mathrm{~Hz}$.

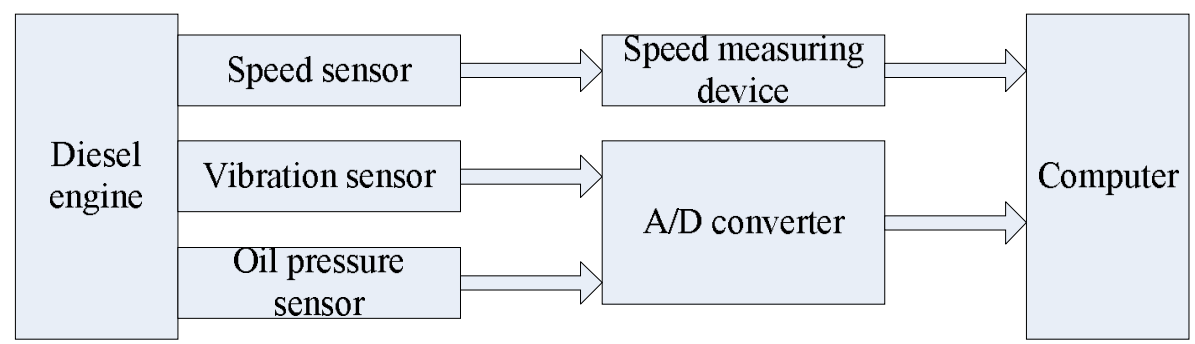

Figure 7. Vibration signal acquisition system.

The vibration signals of the engine under different wear conditions are collected, as shown in Figure 8 and Figure 9. In Figure 8 and Figure 9, there is a large amount of background noise in the vibration signals of different wear conditions of the the crankshaft bearing, which is unfavorable to the extraction of fault features.

(a) Normal wear

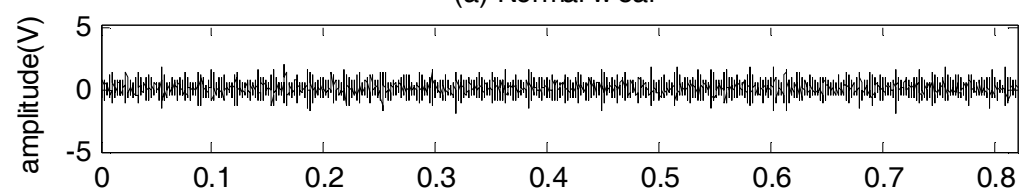

(b) Minor wear

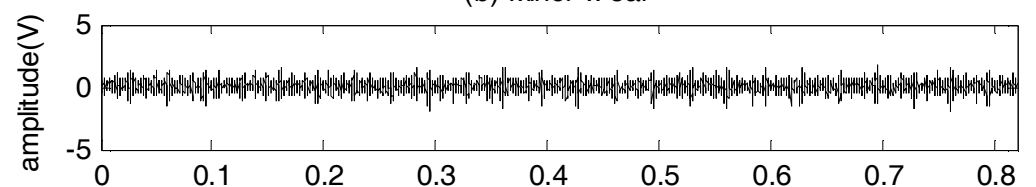

(c) Moderate wear

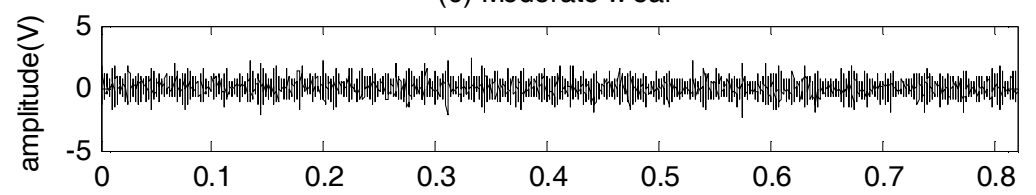

(d) Severe wear

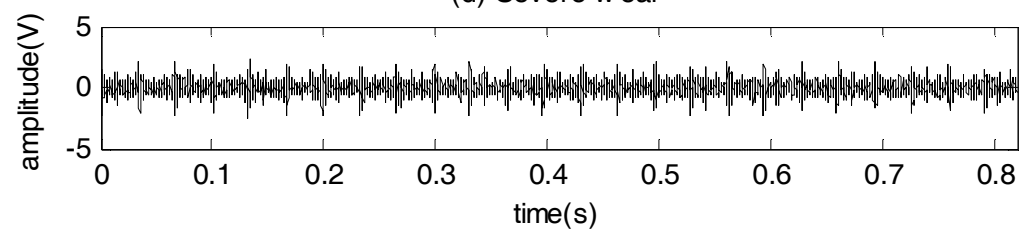

Figure 8. The time domain waveform of vibration signal. 
(a) Normal w ear

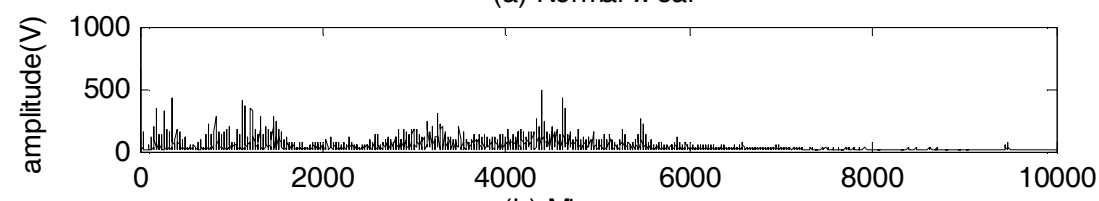

(b) Minor w ear

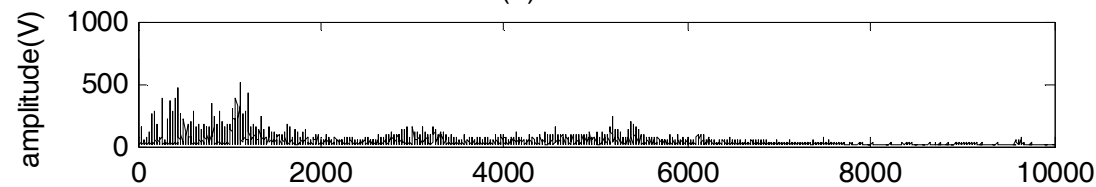

(c) Moderate w ear

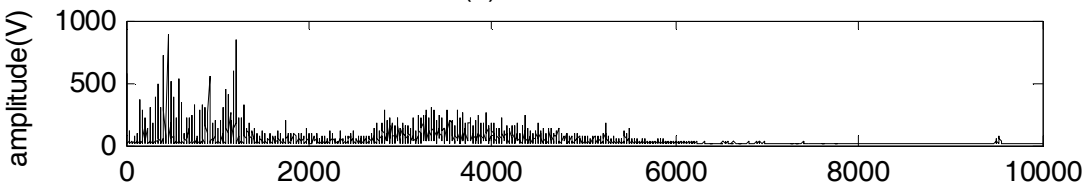

(d) Severe wear

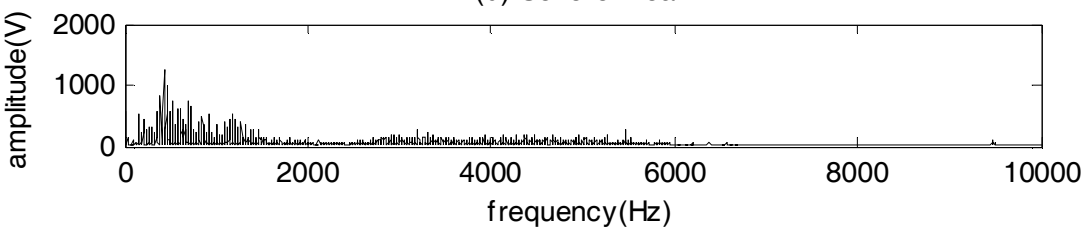

Figure 9. The frequency domain waveform of vibration signal.

\subsection{Experimental Data Processing}

According to the proposed method, the VMD method is used to decompose the vibration signals under different wear conditions. The genetic algorithm is used to determine the number of components [29]. According to the decomposition results of the vibration signals, the permutation entropy of each component under different wear conditions is calculated respectively, as shown in table 4.

Table 4. The permutation entropy of each component under different wear conditions.

\begin{tabular}{ccccc}
\hline Components & Normal wear & Minor wear & Moderate wear & Severe wear \\
\hline U1 & 0.368 & 0.364 & 0.332 & 0.315 \\
U2 & 0.424 & 0.414 & 0.407 & 0.418 \\
U3 & 0.563 & 0.563 & 0.534 & 0.565 \\
U4 & 0.610 & 0.658 & 0.560 & 0.624 \\
U5 & 0.637 & 0.638 & 0.649 & 0.664 \\
\hline
\end{tabular}

As we can see from Table 4, the permutation entropy values of the component U1 under different wear conditions are all the smallest, that is to say, the component $U 1$ contains the most abundant fault information. Therefore, the component U1 is selected as the fault component. The Hilbert envelope spectrums of the component U1 under different wear conditions are calculated respectively, as shown in Figure 10. 
(a) Normal w ear

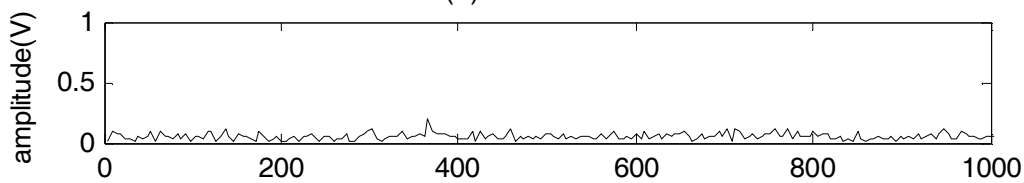

(b) Minor w ear

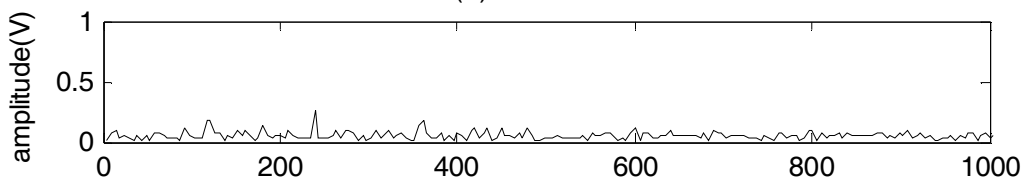

(c) Moderate wear

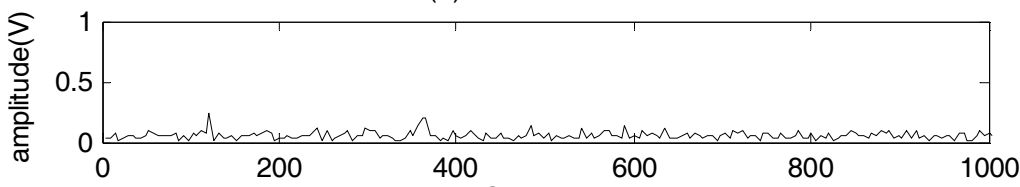

(d) Severe w ear

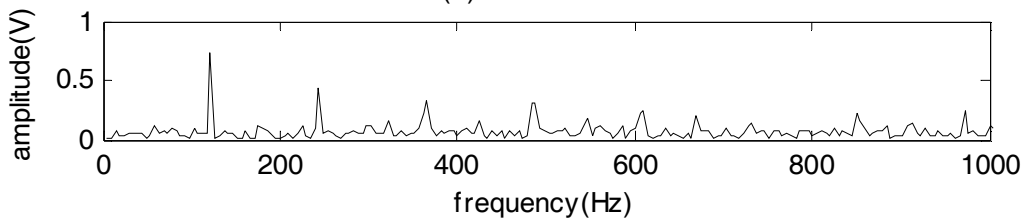

Figure 10. The Hilbert envelope spectrums of the component U1 under different wear conditions.

As can be seen from Figure 10, the characteristic frequency of the fault appears under the severe wear condition, and the characteristic frequency under the other wear conditions is not obvious enough. With the increase of the clearance of the crankshaft, the energy of the characteristic frequency point does not increase gradually. Therefore, the fault component is further processed RDT, and its Hilbert envelope spectrum is shown as shown in Figure 11.

(a) Normal wear

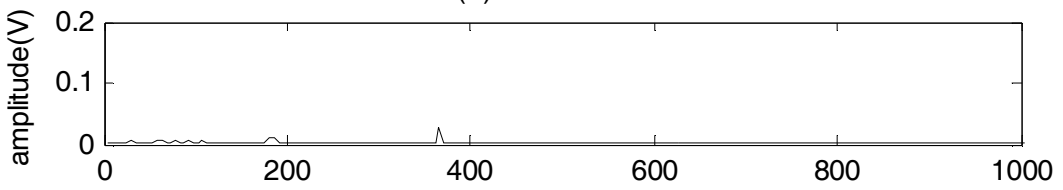

(b) Minor w ear

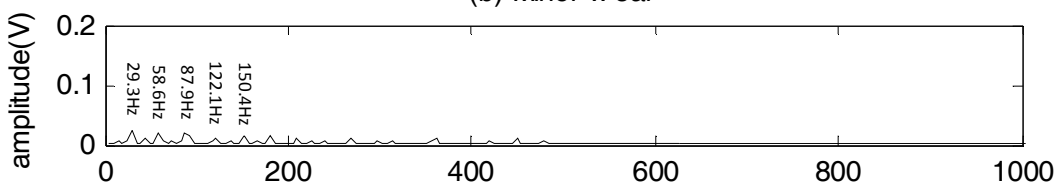

(c) Moderate w ear
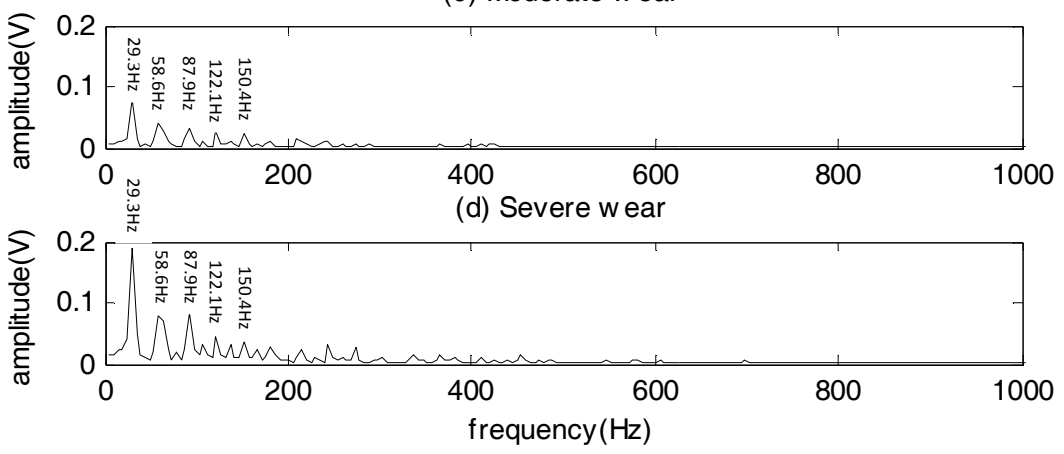

Figure 11. The Hilbert envelope spectrum of the fault components after RDT. 
As can be seen from Figure 11, in the Hilbert envelope spectrum under different wear conditions, the rotation frequency, such as $29.3 \mathrm{~Hz}, 58.6 \mathrm{~Hz}, 122.1 \mathrm{~Hz}, 151.4 \mathrm{~Hz}, 210 \mathrm{~Hz}$ and ignition frequency, such as $87.9 \mathrm{~Hz}$ and $180.7 \mathrm{~Hz}$, are found. With the increase of the clearance of the crankshaft, the energy of the characteristic frequency point increases gradually. In order to further illustrate the effect, the energy of the above characteristic frequency points in the Hilbert envelope spectrum is calculated respectively, as shown in Table 5.

Table 5. The permutation entropy of each component under different wear conditions.

\begin{tabular}{ccccc}
\hline $\begin{array}{c}\text { Characteristic } \\
\text { frequency }(\mathrm{Hz})\end{array}$ & Normal wear & Minor wear & Moderate wear & Severe wear \\
\hline 29.3 & 0.0084 & 0.0230 & 0.0807 & 0.1890 \\
58.6 & 0.0054 & $0 / 0197$ & 0.0402 & 0.0781 \\
87.9 & 0.0083 & 0.0199 & 0.0325 & 0.0828 \\
122.1 & 0.0039 & 0.0115 & 0.0267 & 0.0465 \\
151.4 & 0.0019 & 0.0137 & 00232 & 0.0380 \\
180.7 & 0.0117 & 0.0149 & 0.0178 & 0.0279 \\
210.0 & 0.0013 & 0.0095 & 0.0115 & 0.0252 \\
\hline
\end{tabular}

As can be seen from Table 5, with the increase of the clearance of the crankshaft, the energy of the characteristic frequency point increases gradually, which is in accordance with the fault rule set by the test. The energy of the characteristic frequency point can reflect the vibration intensity of the engine. The greater the clearance of the crankshaft is, the stronger the impact of the engine. Therefore, according to the proposed method, the characteristic frequency of the engine crankshaft bearing fault is successfully extracted.

In order to further verify the advantages of VMD, the signal is processed in a similar way based on EMD and EEMD, as shown in Figure 12 and Figure 13.As can be seen from Figure 12 and Figure 13, fault feature cannot be effectively extracted based on EEMD and EMD, which shows the advantages of the proposed method.

(a) Normal wear
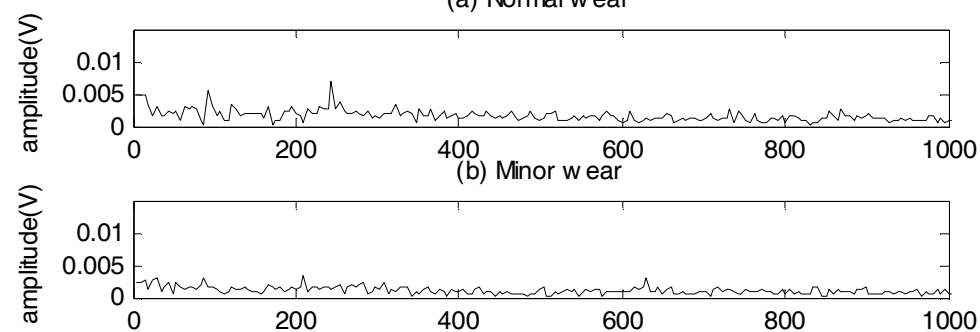

(c) Moderate w ear
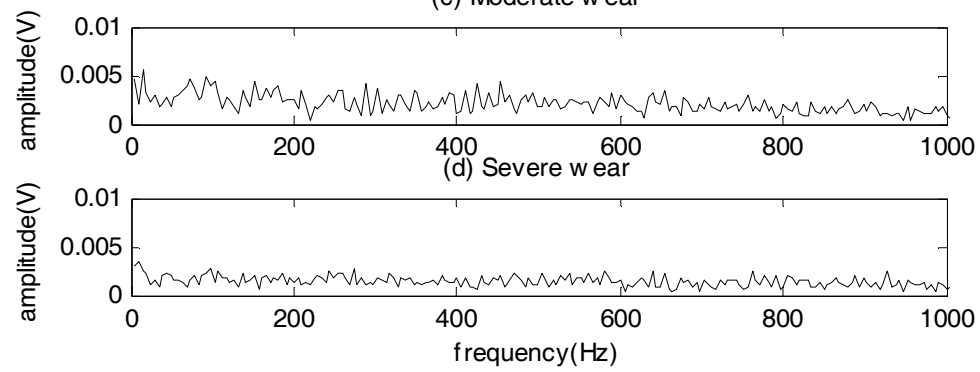

Figure 12. Feature extraction method based on EMD. 
(a) Normal wear
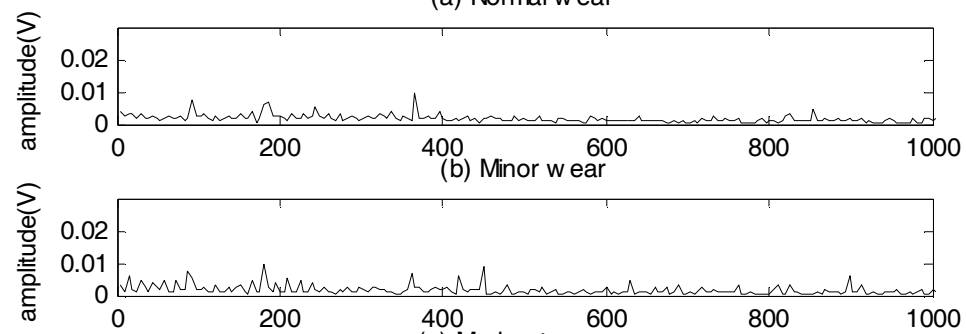

(c) Moderate w ear

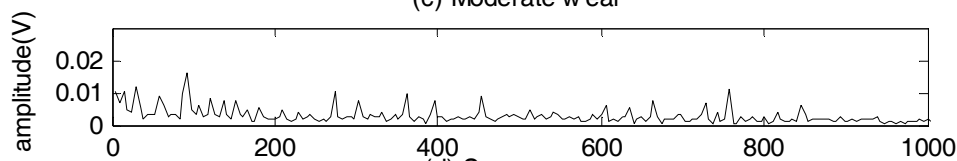

(d) Severe w ear

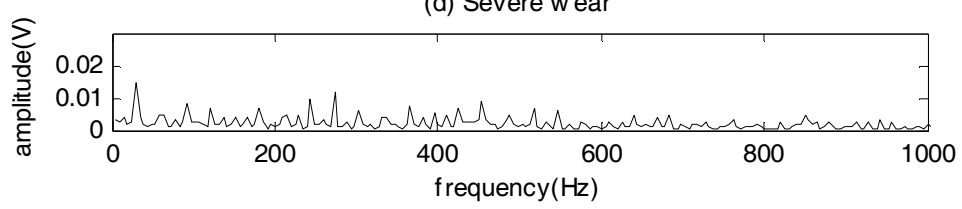

Figure 13. Feature extraction method based on EEMD.

\section{Conclusions}

In the engineering application, the mechanical structure of the engine is very complex, which leads to the complex transmission path of the vibration signal. As a result, the excitation and response of multiple vibration sources are coupled to each other, which make the diagnosis of crankshaft wear fault very difficult. In order to solve the above problems, a fault feature extraction method for engine crankshaft bearing is proposed based on the variational mode decomposition and random reduction technology. The research work of the simulation signal analysis and the fault feature extraction of the crankshaft bearing are carried out. The conclusions are as follows:

(1) The proposed method can overcome the strong background noise, and successfully extract the rotation frequency and ignition frequency of the simulation signal.

(2) The proposed method is applied to the fault diagnosis of engine crankshaft bearing, and the characteristic frequencies of crankshaft bearing fault under different wear conditions are successfully extracted, which shows that the proposed method is effective. In addition, compared with the method based on EMD and EEMD, the feature extraction result of the proposed method is better, which shows that the method is more advanced.

\section{Acknowledgment}

This work was funded by Key Project of the Army Equipment Department (Grant no.WG2015JJ010008).

Author Contributions: Gang Ren conceived of and designed the study. Jiajia Han and Xiangyu Jia were involved in the data collection and experimental work under the supervision of Gang Ren. Gang Ren wrote the paper under the supervision of Jide Jia and Jianmin Mei. Jide Jia reviewed and edited the manuscript. All authors contributed to discussing and revising the manuscript. 


\section{Conflicts of Interest}

The authors declare that there are no conflicts of interest regarding the publication of this paper.

\section{References}

1. Rauber T. W., Boldt F. D. A., et al. Heterogeneous Feature Models and Feature Selection Applied to Bearing Fault Diagnosis. IEEE Transactions on Industrial Electronics. 2015, Vol.62, Issue 1, p. 637646. [CrossRef]

2. Xie Z.J., Song B.Y., Zhang Y., Zhang F. Application of an Improved Wavelet Threshold Denoising Method for Vibration Signal Processing. Advanced Materials Research. 2014, Issue 889-890, p. 799806. [CrossRef]

3. HUANG N. E., SHEN Z., LONG S. R. The empirical mode decomposition and the Hilbert spectrum fornonlinear and non-stationary time series analysis. Proceedings of the Royal Society. A. 1998, Issue 454, p. 903-995.

4. HUANG N. E., SHEN Z., LONG S. R. A new view of non- linear water waves:The Hilbert spectrum. Annu. Rev. Fluid Mech. 1999, Issue 31, p. 417-457.

5. Ai L., Wang J., Yao R. Classification of parkinsonian and essential tremor using empirical mode decomposition and support vector machine. Digital Signal Process.2011, Vol. 21, Issue 4, p. 543550. [CrossRef]

6. SU W.S., Wang F.T., Zhang Z.X., et al. Application of EMD denoising and spectral kurtosis in early fault diagnosis of rolling element bearings. Journal of Vibration \& Shock.2010, Vol. 22, Issue 1, p. 3537-3540.

7. Lahmiri., Boukadoum M. A weighted bio-signal denoising approach using empirical mode decomposition. Biomed. Eng. Lett.2015, Vol. 5, Issue 2, p. 131-139.

8. Huang C, Wang H., Long B. Signal Denoising Based on EMD. IEEE Circuits \& Systems International Conference on Testing \& Diagnosis.2009, p. 1-4.

9. Zhang Y.K., Ma X.C., Hua D.X., et al. An EMD-based denoising method for lidar signal. International Congress on Image \& Signal Processing.2010, Issue 8, p. 4016-4019.

10. Khaldi K., Turki-Hadj Alouane M., et al. A new EMD denoising approach dedicated to voiced speech signals. International Conference on Signals.2008, p. 1-5. [CrossRef]

11. Wang T., Zhang M., Yu Q., et al. Comparing the application of EMD and EEMD on time-frequency analysis of seimic signal. Journal of Applied Geophysics, 2012, Vol. 83, Issue 6, p. 29-34.

12. WU Z. H., HUANG N. E. ENSEMBLE EMPIRICAL MODE DECOMPOSITION: A NOISE-ASSISTED DATA ANALYSIS METHOD. Advances in Adaptive Data Analysis, 2005, Vol. 1, Issue 1, p. 0900004.

13. Wang H., Chen J., Dong G. Feature extraction of rolling bearing's early weak fault based on EEMD and tunable Q-factor wavelet transform. Mechanical Systems \& Signal Processing, 2014, Vol. 48, Issue 2, p. 103-119. [CrossRef] 
14. Dragomiretskiy K., Zosso D. Variational Mode Decomposition. IEEE Transactions on Signal Processing.2013, Vol. 62, Issue 3, p. 531-544. [CrossRef]

15. Zhao C., Feng Z. P. Application of multi-domain sparse features for fault identification of planetary gearbox. Measurement.2017, Issue 104, p. 169-179.

16. An X. L., Tang Y. J. Denoising of hydropower unit vibration signal based on variational mode decomposition and approximate entropy. Transactions of the Institute of Measurement and Control. 2016, Vol. 38, Issue 3, p. 282-292.

17. Li Z. P., Chen J. L., Zi Y. Y. Independence-oriented VMD to identify fault feature for wheel set bearing fault diagnosis of high speed locomotive. Mechanical Systems and Signal Processing. 2017, Issue 85, p. 512-529. [CrossRef]

18. E J. W., Bao Y. L., Ye J. M. Crude oil price analysis and forecasting based on variational mode decomposition and independent component analysis. Physica A.2017, Issue 484, p. 412-427.

19. An X. L., Tang Y. J. Application of variational mode decomposition energy distribution to bearing fault diagnosis in a wind turbine. Transactions of the Institute of Measurement and Control. 2017, Vol. 39, Issue 7, p. 1000-1006.

20. Liu Y. Y., Yang G. L., Li M. Variational mode decomposition denoising combined the detrended fluctuation analysis. Signal Processing.2016, Issue 125, p. 349-364. [CrossRef]

21. Yao J. C., Xiang Y., Qian S. C. Noise source identi- fication of engine based on variational mode decomposition and robust independent component analysis. Applied Acoustics. 2017, Issue 116, p. 184-194. [CrossRef]

22. Cole H. A. On-the-line analysis of random vibration [C] //AIAA /ASME 9th Structure, Structural Dynamics and Materials Conference, AIAA Paper, 1968: 268-288.

23. Cole H. A. Method and apparatus for measuring the damping characteristic of a structure: United State, 3620069[P]. 1971.

24. Mikael A., Gueguen P., Bard P. Y., et al. The Analysis of Long-Term Frequency and Damping Wandering in Buildings Using the Random Decrement Technique. Bulletin of the Seismological Society of America, 2013, Vol. 103, Issue 1, p. 236-246. [CrossRef]

25. Yang J. C. S., Chen. J., Dagalakis N G. Damage detection in offshore structures by the Random Decrement Technique. Journal of Energy Resources Technology, 1984, Vol. 55, Issue 106, p. 637642. [CrossRef]

26. Bandt C., Pompe B. Permutation entropy: a natural complexity measure for time series.. Physical Review Letters, 2002, Vol. 88, Issue 17, p. 174102.

27. Yan. R, Liu Y., Gao R. X. Permutation entropy: A nonlinear statistical measure for status characterization of rotary machines. Mechanical Systems \& Signal Processing, 2012, Vol. 29, Issue 5, p. 474-484. [CrossRef]

28. Xiao, Y., Mei, J., Zeng, R. Mechanical fault diagnose of diesel engine based on bispectrum and Support Vector Machines. IEEE International Conference on Computer Science and Information 
Technology (pp.425-429). IEEE.

29. Li Y., Li H., Wang B., et al. Rolling Element Bearing Performance Degradation Assessment Using Variational Mode Decomposition and Gath-Geva Clustering Time Series Segmentation. International Journal of Rotating Machinery, 2017, Issue 2017, p. 1-12. [CrossRef] 